\title{
Contextual impairments in schizophrenia and the FN400
}

\author{
Lucia Amoruso 1,2, Juan Cardona ${ }^{1,2,3}$, Margherita Melloni' ${ }^{1}$, Lucas Sedeño ${ }^{1}$ and Agustin Ibanez 1,2,3,4* \\ ' Laboratory of Experimental Psychology and Neuroscience, Institute of Cognitive Neurology, Buenos Aires, Argentina \\ 2 National Scientific and Technical Research Council, Buenos Aires, Argentina \\ 3 Institute of Neuroscience, Favaloro University, Buenos Aires, Argentina \\ ${ }^{4}$ Laboratory of Cognitive and Social Neuroscience, Universidad Diego Portales, Santiago, Chile \\ *Correspondence: agmabaib@gmail.com
}

Edited by:

John J. Foxe, Albert Einstein College of Medicine, USA

Reviewed by:

Walter Ritter, Nathan Kline Institute for Psychiatric Research, USA

Pierfilippo De Sanctis, Albert Einstein College of Medicine, USA

\section{A commentary on}

ERP investigation of study-test background mismatch during face recognition in schizophrenia

by Guillaume et al. (2012). Schizophr. Res. 134, 101-109.

Our brains are good at extracting and processing social contextual cues. Ongoing information is rapidly linked to memory traces of previous experiences, allowing us to generate predictions which help us interpret daily situations. These predictions are a core aspect of human cognition as long as they make social behaviors more efficient. Current evidence, however, suggests that schizophrenia patients are less able to benefit from context and, consequently, social impairments are commonly observed in this complex disorder.

Over the last decades, event-related potentials (ERPs) have been used to investigate the electrophysiological correlates of several memory processes. In classical explicit memory experiments, familiarity (the subjective experience of knowing an item - e.g., a face-without being able to retrieve any further episodic information) has been associated with a mid-frontal old/new difference occurring in the 300-500 ms window, often called "FN400" (Rugg and Curran, 2007). For example, the comparison of ERPs on hits and correct rejections (old/new effects) during face recognition, lead to higher FN400 amplitudes for correctly rejected new items than for correctly recognized old ones. Importantly, this difference is observed despite recognition is followed by the recollection of specific details associated to the item (e.g., occupations asso- ciated with the faces, Curran and Hancock, 2007). This lack of ability in retrieving episodic details is attributed to the fact that it is perceived out of the context in which is commonly embedded.

Recently, Guillaume et al. (2012) have investigated FN400 old/new effects in schizophrenia during face recognition. The aim of their study was to evaluate if familiarity was affected by contextual information mismatching in schizophrenia patients versus controls by manipulating the background in which faces appeared. This resulted in three conditions: old (faces that were presented on the study phase with the same background), different (faces that were presented on the study phase with a different background), and new condition (faces that were not presented on the study phase). They found that, across conditions, schizophrenia patients showed greater FN400 amplitude compared to controls. Moreover, in the patients, the FN400 amplitude was the same in the different and new conditions suggesting that familiarity was severely disrupted when the context in which faces appeared was changed.

Although correlates of familiarity have been classically linked to the FN400 (Rugg and Curran, 2007), recent work (Voss et al., 2012) has called into question this traditionally accepted linkage, suggesting that modulations in this component could be actually indexing implicit conceptual memory processing instead of familiarity per se. It is important to note that the " $F$ " in FN400 indicates a more frontal topographical distribution compared to the similar N400 component found for words semantically anomalous to the preceding context. While this difference in the scalp activity is consistent with the existence of distinct roles for each negativity (FN400 in familiarity versus
N400 in language), it has been suggested (Kutas and Federmeier, 2011) that the classical N400 and the FN400 could be indexing an analog process.

In our view, both proposals are justified and can explain, in a more parsimonious fashion, current FN400-related data. However, based on the possible functional analogy between the FN400 and the N400 and also on current neuroanatomical evidence suggesting frontal and temporal sources (Halgren et al., 2002; Voss et al., 2008; Ibanez et al., 2012), we propose that this component might be reflecting a more general process regarding implicit contextual facilitation.

We have recently proposed that the N400 reflects the activation of a contextual frontotemporal network responsible for the instantiation of meaning construction under the crucial role of context-based information (Amoruso et al., 2011; Ibanez and Manes, 2012). In our model, similar to the one proposed by Bar (2004) for object recognition, frontal regions would play a key role in updating ongoing contextual information in working memory and integrating it with semantic knowledge about target-context associations and their learning value stored in temporal regions. Taken together, information about current and previous experiences is used by the brain in order to make focused predictions to facilitate the interpretation of the ongoing situation.

Furthermore, our model is supported by current experimental evidence from electrocorticography $(\mathrm{ECoG})$ direct recordings (Ibanez et al., 2012) and source estimation (Halgren et al., 2002) which suggests that the scalp-recorded N400 reflects the coordinated activity of multiple brain areas, 
including temporal and frontal regions. Importantly, these two regions, also thought to be involved in the FN400 generation (Voss et al., 2012), are the most affected in schizophrenia, at structural, and functional level (Wong and Van Tol, 2003).

Contextual deficits seem to be the core of N400 abnormal modulations found in schizophrenia (Kumar and Debruille, 2004; Ibáñez et al., 2011). Moreover, contextual impairments seem to be present in this condition, from the basic perceptual level (Chambon et al., 2008) to the higher aspects of social cognition (Chung et al., 2011). FN400 abnormal modulations in schizophrenia patients found by Guillaume et al. (2012) can be explained as a disruption in the integration of contextual cues in the frontotemporal network. More specifically, the greater FN400 amplitude showed by these patients compared to controls suggests that they are not able to benefit from context, maybe due to a reduction in functional connections between frontal and temporal lobes. This aforementioned disruption can be more specifically understood in terms of an inability to maintain a representation of context in working memory (Cohen and Servan-Schreiber, 1999).

In this view, the FN400 component would indexes a more general and constructive process regarding implicit context-based facilitation in the brain, a process that is severely disrupted in schizophrenia. By this means, our approach would be able to explain not only normal old-new FN400 effects and N400 classic modulations, but also schizophrenia abnormal ones, providing a broader and unified framework to interpret the N400 findings and the contextual impairments observed in this complex disorder.

\section{ACKNOWLEDGMENTS}

This work was supported by CONICET and INECO Foundation grants.

\section{REFERENCES}

Amoruso, L., Couto, B., and Ibanez, A. (2011). Beyond extrastriate body area (EBA) and fusiform body area (FBA): context integration in the meaning of actions. Front. Hum. Neurosci. 5:124. doi: 10.3389/ fnhum.2011.00124

Bar, M. (2004). Visual objects in context. Nat. Rev. Neurosci. 5, 617-629.

Chambon, V., Franck, N., Koechlin, E., Fakra, E., Ciuperca, G., Azorin, J. M., and Farrer, C. (2008). The architecture of cognitive control in schizophrenia. Brain 131, 962-970.

Chung, Y. S., Mathews, J. R., and Barch, D. M. (2011). The effect of context processing on different aspects of social cognition in schizophrenia. Schizophr. Bull. 37, 1048-1056.

Cohen, J. D., and Servan-Schreiber, D. (1999). Context, cortex, and dopamine: a connectionist approach to behavior and biology in schizophrenia. Psychol. Rev. $99,45-77$.

Curran, T., and Hancock, J. (2007). The FN400 indexes familiarity-based recognition of faces. Neuroimage 36, 464-471.

Guillaume, F., Guillem, F., Tiberghien, G., and Stip, E. (2012). ERP investigation of study-test background mismatch during face recognition in schizophrenia. Schizophr. Res. 134, 101-109.

Halgren, E., Dhond, R. P., Christensen, N., Van, P. C. Marinkovic, K., Lewine, J. D., and Dale, A. M. (2002). N400-like magnetoencephalography responses modulated by semantic context, word frequency, and lexical class in sentences. Neuroimage 17, 1101-1116.

Ibanez, A., Cardona, J. F., Vidal Dos Santos, Y., Blenkmann, A., Aravena, P., Roca, M., and Bekinschtein, T. (2012). Motor-language coupling: direct evidence from early Parkinson's disease and intracranial cortical recordings. Cortex. doi: 10.1016/j.cortex.2012.02.014

Ibanez, A., and Manes, F. (2012). Contextual social cognition and the behavioral variant of frontotemporal dementia. Neurology 78, 1354-1362.

Ibáñez, A., Riveros, R., Aravena, P., Vergara, V., Cardona, J. F., García, L., Hurtado, E., Reyes, M. M., Barutta, J., and Manes, F. (2011). When context is difficult to integrate: cortical measures of congruency in schizophrenics and healthy relatives from multiplex families. Schizophr. Res. 126, 303-305.

Kumar, N., and Debruille, J. B. (2004). Semantics and N400: insights for schizophrenia. J. Psychiatry Neurosci. 29, 89-98.

Kutas, M., and Federmeier, K. D. (2011). Thirty years and counting: finding meaning in the N400 component of the event-related brain potential (ERP). Annu. Rev. Psychol. 62, 621-647.

Rugg, M. D., and Curran, T. (2007). Event-related potentials and recognition memory. Trends Cogn. Sci. (Regul. Ed.) 11, 251-257.

Voss, J. L., Lucas, H. D., and Paller, J. L. (2012). More than a feeling: pervasive influences of memory processing without awareness of remembering. Cogn. Neurosci. doi: 10.1080/17588928.2012.674935

Voss, J. L., Reber, P. J., Mesulam, M. M., Parrish, T. B., and Paller, K.A. (2008). Familiarity and conceptual priming engage distinct cortical networks. Cereb. Cortex 18, 1712-1719.

Wong, A. H., and Van Tol, H. H. (2003). Schizophrenia: from phenomenology to neurobiology. Neurosci. Biobehav. Rev. 27, 269-306.

Received: 29 April 2012; accepted: 08 June 2012; published online: 02 July 2012.

Citation: Amoruso L, Cardona J, Melloni M, Sedeño L and Ibanez A, (2012) Contextual impairments in schizophrenia and the FN400. Front. Hum. Neurosci. 6:191. doi: 10.3389/ fnhum.2012.00191

Copyright (C) 2012 Amoruso, Cardona, Melloni, Sedeño and Ibanez. This is an open-access article distributed under the terms of the Creative Commons Attribution Non Commercial License, which permits non-commercial use, distribution, and reproduction in other forums, provided the original authors and source are credited. 